\title{
An Accurate Multi-Biometric Personal Identification Model using Histogram of Oriented Gradients (HOG)
}

\author{
Mostafa A. Ahmad ${ }^{1,2}$, Ahmed H. Ismail ${ }^{1,3}$, Nadir Omer ${ }^{1}$ \\ ${ }^{1}$ College of Computing \& Information Technology, University of Bisha, Bisha, Saudi Arabia \\ ${ }^{2}$ Faculty of Computers \& Information, Menofia University, Shibeen El-Kom, Egypt \\ ${ }^{3}$ Faculty of Science, Menofia University, Shibeen El-Kom, Egypt
}

\begin{abstract}
Biometrics is the detection and description of individuals' physiological and behavioral features. Many different systems require reliable personal identification schemes to either prove or find out the identity of an individual demanding their services. Multi-biometrics are required inside the current context of large worldwide biometric databases and to provide new developing security demands. There are some distinctive and measurable features used to distinguish individuals known as Biometric Identifiers. Multi-biometric systems tend to integrate multiple identifiers to increase recognition accuracy. Face and digital signature identifiers are still a challenge in many applications, especially in security systems. The fundamental objective of this paper is to integrate both identifiers in an accurate personal identification model. In this paper, a reliable multi-biometric model based on Histogram of Oriented Gradients (HOG) features of a face and digital signature and is able to identify individuals accurately is proposed. The methodology is to adopt many parameters such as weights of HOG features in merging process, the HOG parameters itself, and the distance method in matching process to gain higher accuracy. The proposed model achieves perfect results in personal identification using HOG features of digital signature and face together. The results show that the HOG feature descriptor significantly performs target matching at an average of $100 \%$ accuracy ratio for face recognition together with the digital signature. It outperforms existing feature sets with an accuracy of $\mathbf{8 4 . 2 5 \%}$ for face only and $\mathbf{9 7 . 4 2 \%}$ for digital signature only.
\end{abstract}

Keywords-Biometric identifiers; personal identification; multibiometric systems; face recognition; digital signature; Histogram of Oriented Gradients (HOG)

\section{INTRODUCTION}

Biometrics refer to personality check of people as indicated to their physical or behavioral qualities [1]. Biometric technology is a technology that allows people to have an digiticaly authentication using different parts of their physical bodies [2]. Numerous physical body parts and individual highlights have been utilizing for biometric Systems: faces, digital signatures, and DNA. Person verification in view of biometric highlights has pulled in more consideration in planning security systems [3]. The spread of biometrics is enacted by two parts: technical specialized and the necessity for security. Biometric is critical to keep data secured in our day by day life [4], [5]. The requirement for unique sensors to acquire biometrics was for quite some time thinking about disadvantages, particularly if multi-biometrics was considered [5]. Nonetheless, no single biometrical feature can meet all the execution necessities in reasonable systems. Face recognition has as of late gotten critical consideration. It assumes a vital part in numerous application regions, for example, humanmachine communication, verification, and surveillance. Digital signature are recognized by current standards and legislation as a term that use a key pair of user for sign and verify a document using biometric systems [2], [6]. Today there are many advantages for digital signature such as offers more security than any electronic signature, independent verification cannot be alter by unauthorized parties and long-term retention and access. Face recognition and digital signature have been a long-standing issue in PC vision [7]. As of late, Histograms of Oriented Gradients (HOGs) have turned out to be an effective descriptor as feature extraction for object recognition in general and face recognition and digital signature in particular. Face and digital signature have been a long-standing problem in many applications, especially in computer vision. The main contribution of this paper is to integrate both identifiers in an accurate personal identification model. The proposed multibiometric model is based on HOG features of a face and digital signature and this model able to identify individuals accurately.

This paper is organized as follows. The related work is describes in Section 2, as well as our topic and techniques. Section 3 introduces the HOG descriptor. In Section 4, we describe the methodology of our multi-biometric system with its different modules. Section 5 explains the description of the dataset used in the testing process as well as the results and experimental analysis. Finally, the main conclusion is drawn in Section 6 with a hint for the future work we have engaged in follow-up this work.

\section{RELATED WORK}

Biometrics deals with innovations used of gauge human physical or behavioral characteristics to distinguish and perceive people [8]. For the of biometric there are two types features: physiological (e.g. iris, face, unique mark) and behavioral (e.g. voice and digital signature) [9]. The mix of biometric systems, otherwise called "biometric fusion", can be ordered into unimodal biometric in the event that it depends on a single biometric characteristic and multimodal biometric in the event that it utilizes a few biometric qualities for individual verification [8]. A few systems and structures identified with 
the mix of biometric systems, both unimodal and multimodal is examine and grouped by a given scientific classification. Face recognition is the most broadly recognized people most of the time by recognizing the face of the individuals and advancement in computing skill over the past few decades [10]. There are three stages namely face detection, feature extraction and face recognition in face recognition system [11]. Techniques for face identification and recognition frameworks can be influence by posture, nearness or nonattendance of auxiliary segments, outward appearance, impediment, picture introduction and imaging conditions. It is difficult to implement a strong face recognition framework, which work in all condition. In computer vision, Face recognition has been a long-standing problem. Recently face recognition system attracted significant attention due to the accessibility of inexpensive digital cameras and computers, and its different applications in biometrics and surveillance [12]. In any case, the wide-run varieties of a human face, because of stance, brightening, and demeanor, result in an exceedingly complex appropriation and fall apart the acknowledgment execution. What's more, the issue of machine recognition of human faces keeps on pulling in scientists from orders, for example, pattern recognition and digital signature [10]. First, the Face recognition system detects the presence of a face in an image. If is found, the system's role is to trace the position of one or more faces in the image.

To make robust use for face recognition, O. Deniz [12] in his study investigated a powerful approach based on HOG features. The use of HOG for face recognition and he used the HOG to extract features from overlapping cells because it is important for this case. Also, applied four databases in the study and obtaining significant result based on FERER database. Also in his paper Alberto [6] proposed HOG-EBGM for face recognition. He used $\mathrm{HOG}$ descriptor with three databases and FERET is one them and he obtained better performance by change the properties of HOG to get maximum accuracy of the face graphs acquired compared to classical Gabor-EBGM ones. In their research Bin li [2] introduce LSHOG for face recognition to extract features based on reduce the dimension of the features compared with HOG idea, which distributed an image into several cells, and computed a histogram of gradient orientations over each cell. Unlike traditional HOG their proposed LSHOG tell a histogram over gradient orientations atop the complete photo at each pixel location. Experimental outcomes confirm the feasibility and efficiency of LSHOG and their face recognition method.

Now a days, Digital signature is a popular term that uses a key pair of user for sign and authentication of a document. Using biometric technology professionals can create their digital signature. Biometric technology is a technology that allows people to have an digiticaly authentication using different parts of their bodies [13]. Mustafa [14] proposed an offline signature verification system based on a signature's local histogram features using classifiers combination of HOG and histogram of local binary patterns (LBP) features. The combination of all classifiers (global and user-dependent classifiers trained with each feature type), achieves a $15.41 \%$ equal error rate in skilled forgery test, in the GPDS-160 signature database without using any skilled forgeries in training. The signature might change over some undefined period and are impacted by physical and enthusiastic states of a subject. The signature may change over some vague time span and are affected by physical and excited conditions of a subject. Further, capable falsifiers may have the ability to imitate signatures that trap the structure Due to outer assembling imperatives in detecting innovations and also innate confinements inside each biometric, no single biometric technique to date can warrant a $100 \%$ verification exactness and utilization independent from anyone else [5], [14], [15]. These frameworks are additionally ready to meet the strict execution prerequisites forced by different applications. In [10], 1-median filtering as a spoofing-resistant summed up contrasting option to the entirety administer focusing on the issue of fractional multi-biometric spoofing where $m$ out of $n$ biometric sources to be joined are attacked. Section 3 introduces the HOG.

\section{HISTOGRAM ORIENTED GRADIENT (HOG)}

The histogram of situated angles was proposed for the utilization of person on pedestrian detection [7]. HOG is a feature extraction strategy that figures the situated gradients of a picture utilizing angle finders. Due to its victories, it has been utilized as a part of numerous PC vision frameworks [1]. For example, it has been utilize for face and on-street vehicle identification applications. It has been connect to face recognizable proof and in addition feeling and gesture recognition. Applicable descriptors assume a critical part in face parameterization. Wavelet, contour lets, and Gabor wavelets have been generally utilize for face recognition. Different parameters like example arranged edge greatness POEM utilizing nearby twofold example LBP and histogram of situated angle HOG have been as of late connect to human location and face recognition. The utilization of introduction histograms has numerous forerunners. Freeman and Roth utilized introduction histograms for hand signal recognition. Histogram of Oriented Gradients (HOG) is highlight descriptors that were first presented by Dalal and Triggs in their CVPR paper [1] to distinguish people on foot in pictures. The examination was then extended to identify human in recordings and creatures and questions in static pictures [16]. In this work, HOG descriptors are extracted to recognize the area of target appearance in face and digital signature images [17]. Fig. 1 shows an image that divided into equal size cells of size $8 \times 8$ pixels. Moreover, each cell is initialized with a 9-bin histogram range from 0 to 180 degrees or 0 to 360 degrees. The magnitude and orientation of each pixel are calculated using (1) and (2), where $G_{x}$ and $G_{y}$ are the horizontal and vertical gradient, respectively.

Magnitude, $|G(x, y)|=\sqrt{G_{x}(x, y)^{2}+G_{y}(x, y)^{2}}$
Orientation, $\tan (\varnothing(x, y))=\frac{G_{y}(x, y)}{G_{x}(x, y)}$

Consequent to getting the introduction and result, every pixel will vote to the 9-bin histogram similarly to its orientation [9]. The quantity of voting will be chosen by its relative size. Therefore, more grounded sizes will largely affect the histogram. By arranging the extent and direction of every cell into a histogram, we are decreasing the gradient mechanisms down to a vector of only 9 values, which are the sum of sizes 
of each bin. In other words, the gradient histogram quantifies the components of every cell in the picture. It is additionally vital to realize that HOG does not keep the data about the gradient or edge positions, but the dissemination of neighborhood power inclination or edge headings.

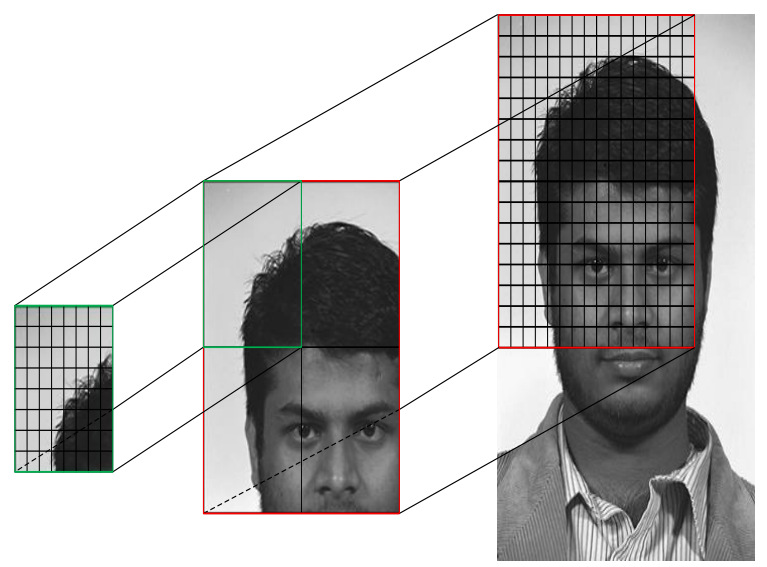

Fig. 1. Normalized face and its spatial cell by HOG descriptor.

Considering that slope is generally affected by brightening changes, standardization is expected to deal with this issue. Instead of normalizing every histogram separately, the cells are first collect into pieces and standardization in view of the considerable number of histograms in the block. In Dalal and Triggs, any block is work of $2 \times 2$ cells as shown in Fig. 1, whereby each blocks cover by half. The histograms of the four cells inside a block are linked into a vector with 36 parts (4 histograms x 9 bins for each histogram) and after that gap this vector by its greatness to normalize it. The most common block normalization used is L2-normalization, as denoted in (3).

$$
f=\frac{V}{\sqrt{\|V\|_{2}^{2}+e^{2}}}
$$

To decrease the calculation time of removing HOG descriptors by moving the window overall GPR picture, the concentration of recognition is limited to the areas that contain potential target reflection. We additionally control the measure of the picture so hyperbolas can be recognizing at various scales. This is vital as the span of hyperbolas shifts in like manner to a few viewpoints like the measurement of the objective, nature of the medium and the setting of the face and signature system itself.

In our work, each spatial cell is square of $8 \times 8$ pixels. This size is selected based on the distance between eyes of the normalized faces, which in our work is 32 pixels and also in our work we used different values to get our result such as the number of bins is choose with different values 9,12 and 15 also the block size is 1, 2, 4 and 8. Finally, the cell size also they have different values like 4, 8, 12 and 16 .

\section{Methodology OF OUR Multi-Biometric SySTEM}

Different Multi-biometric systems share a public general flow as shown in Fig. 2, which is described the four main mechanisms:

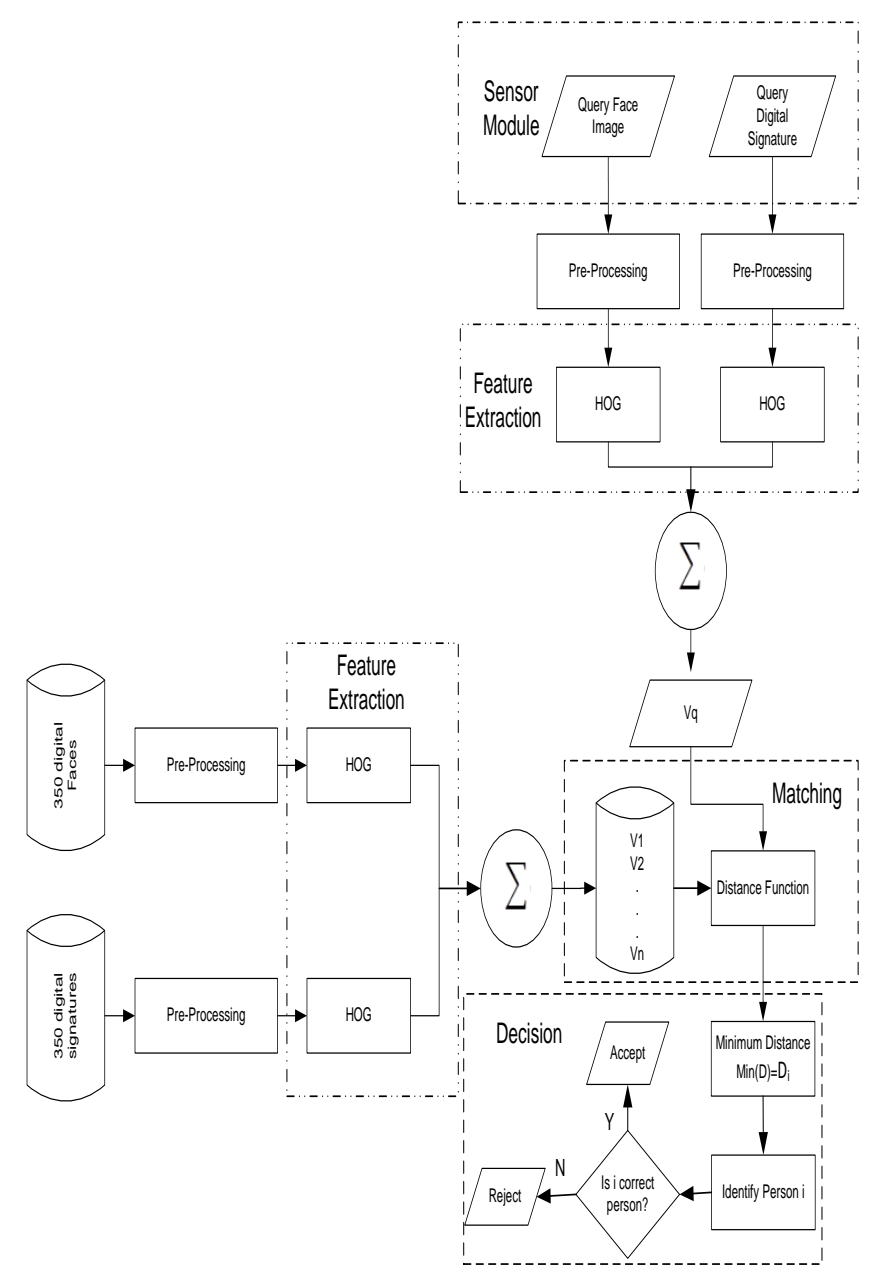

Fig. 2. Block Diagram of suggested multi-biometric system.

- Sensors: The first segment of a multi-biometric system is obtaining of the biometrics information of a person from biometric sensors hardware. For face recognition and digital signature, the sensor is regularly a camera, the sensor is commonly a scanner, for face information, the sensor is a mouthpiece. The nature of the procurement module has a significant effect on the execution of the system that is delicate to the ecological conditions (i.e. changes in the brilliance of a picture), nature of sensor (i.e. dpi of the picture), human factor (i.e. posture varieties).

- Feature extraction module: The gain information is preprepared to expel commotion or different anomalies present and afterward subjected to the component extraction process with a specific end goal to extricate biometrical values that in a perfect world must depict extraordinarily an individual, so biometric information gathered from one individual, under various circumstances, are "comparable", while those gathered from various people are "desperate". For instance, the position and introduction of particulars focus in a fingerprint picture are utilized as a part of a fingerprint framework. The highlights removed amid enlistment are put away in a format, which is a potent little and 
simple to process. Keeping in mind the end goal to enhance interoperability among various biometric frameworks there exist recommendations of the standard configuration of layouts, i.e. for fingerprint they are constructed just with respect to particulars focuses.

- Matching module: In this module, which is not used during enrollment, the feature values from an unknown individual are compared to those in the stored template by generating a matching score which shows the level of similarity between a set of biometrics data. The score should be good enough for features from the same individuals and unacceptable for those from different ones. In a signature system, for example, return the number of matching minutiae points between the query and the template can as a matching score. Usually matching is a difficult pattern-recognition problem due to large intra-class variations (caused by bad acquisition, noise, varying environmental conditions, alterations, etc.) and large inter-class resemblance (i.e. differentiating identical twins is always difficult in face recognition).

- Decision component: In this module, the user's identity is established (identification) or a claimed identity is accepted/rejected based on the matching score. Usually, the final decision is taken by comparing the matching score to a fixed level, which is selected according to consideration of the degree of security required by the application.

The methodology of this paper is to study previous systems in personal identification using multi-biometrics then select the most suitable biometric identifiers such HOG to use multiple identifiers for person identification, finally Measure the accuracy of the developed algorithm according to standard and real data sets. Verify the ability of the developed algorithm to work in real-time.

\section{EXPERIMENTAL RESULTS AND ANALYSIS}

In this section, we present the database of faces and a digital signature, which our experiments are, taking place. After that, we discuss the primary results of our multibiometric identification system.

\section{A. Datasets}

Training and testing of a biometric system dataset plays a main role in achieving better recognition performance. All the experimentations dataset carried out in this paper using two set of images. The first one we got sample dataset for faces from the Face Recognition Technology (FERET) program. The FERET database is a standard database of face imagery which was necessary to evaluate the FERET program, both to supply standard imagery to the algorithm developers and to supply a sufficient number of images to allow testing of these algorithms [9]. FERT dataset contains 3365 full frontal facial images of nearly 1000 subjects. FERT dataset images are organized into a gallery set (fa) and four probe sets ( $\mathrm{fb}, \mathrm{fc}$, dup1, dup2). The second dataset we added some from local images on the way to build our face database from individuals in Bisha University. Our dataset may contain color or grayscale images with different resolutions and file formats. Our experiment is considered 50 persons with 7 face images for each person, a total of 350 faces. The seven samples for each person contain full frontal face views, head rotation, different emotions, and even different clothes or background as shown in Fig. 3. The offline signature database consists of 160 individuals' signatures: each individual has 7 genuine signatures, and 7 forgeries of his signature. The 7 genuine samples of each finger were collected in a single writing session. All signatures have binary bitmap picture format, with 300 dpi resolution, as shown in Fig. 4.

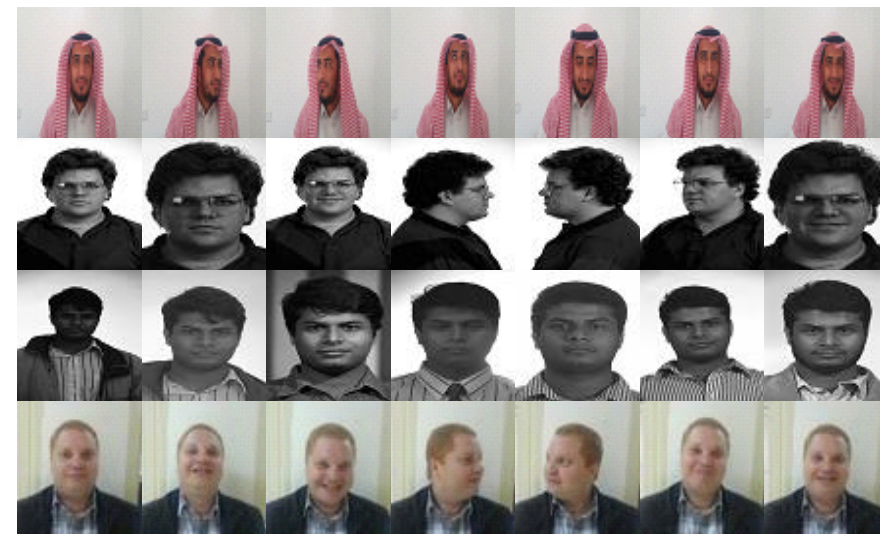

Fig. 3. A sample of faces dataset.

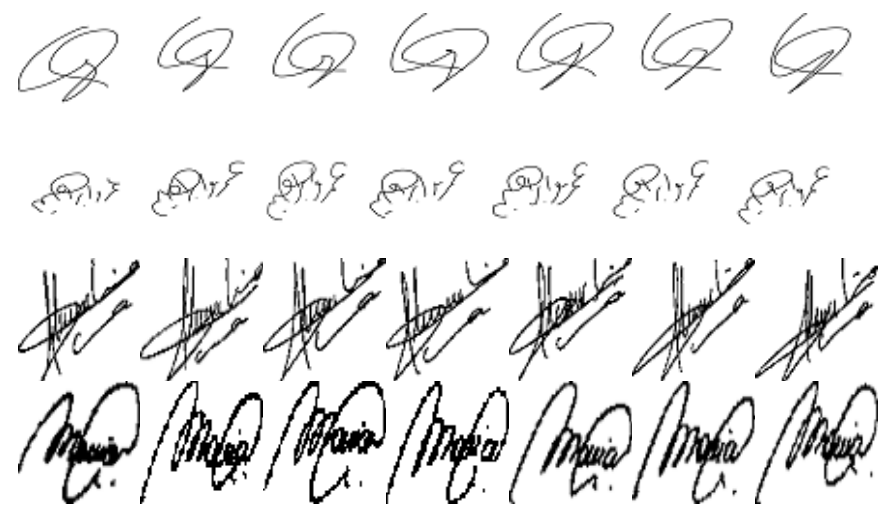

Fig. 4. Sample of digital signature dataset.

\section{B. Experimental Results}

We start our experiment by examining the HOG feature extractor and descriptor on faces and digital signature separately. Preliminary results for faces only were not encouraging, while it ranges between $80 \%$ and $84 \%$ of identification accuracy; the digital signature was ranged between $96 \%$ and $98 \%$. Since we propose to use multibiometric in order to increase the identification accuracy, so we combine both of face and digital signature together to produce a single HOG feature vector $f$ as a weighted sum as shown in (4):

$$
f=\propto f_{1}+(1-\propto) f_{2}
$$

Where, $0 \leq \alpha \leq 1, f_{1}$ is the HOG feature vector for the face, $f_{2}$ is the HOG feature vector for the digital signature. 
To measure the matching between feature vectors of faces or digital signatures, we use some of the distance functions. Some most popular distance functions are Manhattan, Euclidean, Angle-Based, and modified Manhattan. To show the concept of different functions, let $\mathrm{x}$ and $\mathrm{y}$ be two feature vectors of length $\mathrm{n}$, then we can calculate the following distances between these feature vectors as in the following (5)(8):

Manhattan distance:

$d(x, y)=L_{p=1}(x, y)=\sum_{i=1}^{n}\left|x_{i}-y_{i}\right|$

Euclidean distance:

$d(x, y)=L_{p=2}(x, y)=\sqrt{\sum_{i=1}^{2}\left(x_{i}-y_{i}\right)^{2}}$

Angle - based distance:

$d(x, y)=-\cos (x, y)$

Where,

$$
\cos (x, y)=\frac{\sum_{i=1}^{n} x_{i} y_{i}}{\sqrt{\sum_{i=1}^{n} x_{i}^{2} \sum_{i=1}^{n} y_{i}^{2}}}
$$

Modified Manhattan distance:

$$
d(x, y)=\frac{\sum_{i=}^{n}\left|x_{i}-y_{i}\right|}{\sum_{i=1}^{n}\left|x_{i}\right| \sum_{i=1}^{n}\left|y_{i}\right|}
$$

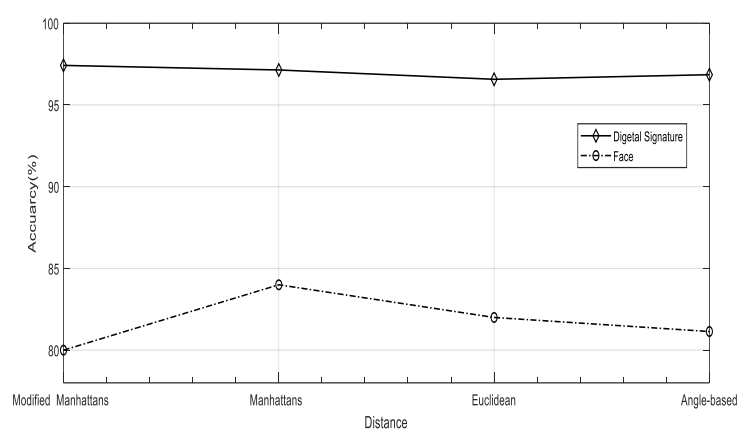

Fig. 5. Performance comparison of HOG among the different distance functions for face and digital signature features in accuracy ratio of identifying.
In Fig. 5, the digital signature identification has outperformed the face in the accuracy ratio all over the distance functions. This is because of the different challenges in face feature detection and description. The accuracy ratio of identification is maximized by the Manhattan distance function in both the face and digital signature. It produces $96.99 \%$ in the digital signature, and $81.78 \%$ in the face on average. In order to achieve better accuracy in individual identification, and benefit from both face and digital signature features, we proposed to merge the two feature vectors of the face and digital signature using different weights according to (4). The results of comparing the accuracy ratios among as shown in Table I, different distance functions at different $\propto$ values (from 0.01 to 0.1 ). It is shown that the Manhattan distance function is outperformed the other functions for all the values of parameter $\propto$. The accuracy ratio is at its maximum value $(99.43 \%)$ at $\propto=$ 0.06 for the Manhattan distance function، which means less weight for the face features in comparing with the digital signature features. The nearest distance function to Manhattan matching accuracy is the modified Manhattan function.

TABLE. I. THE ACCURACY RATIOS IN PERCENTAGE FOR DIFFERENT MATCHING DISTANCES AND VARIOUS $\propto$ WITH A NUMBER OF HOG BINS

\begin{tabular}{|c|c|c|c|c|c|c|c|c|c|c|}
\hline 然 & 0.01 & $\mathbf{0 . 0 2}$ & $\mathbf{0 . 0 3}$ & 0.04 & 0.05 & 0.06 & 0.07 & 0.08 & 0.09 & 0.1 \\
\hline $\begin{array}{c}\text { Modified } \\
\text { Manhatt } \\
\text { an }\end{array}$ & $\begin{array}{c}97.4 \\
3\end{array}$ & $\begin{array}{c}97.7 \\
1\end{array}$ & $\begin{array}{c}98.5 \\
7\end{array}$ & $\begin{array}{c}98.8 \\
6\end{array}$ & $\begin{array}{c}99.1 \\
4\end{array}$ & $\begin{array}{c}98.8 \\
6\end{array}$ & $\begin{array}{c}98.8 \\
6\end{array}$ & $\begin{array}{c}98.2 \\
9\end{array}$ & $\begin{array}{c}98.2 \\
9\end{array}$ & $\begin{array}{c}97.7 \\
1\end{array}$ \\
\hline $\begin{array}{l}\text { Manhatt } \\
\text { an }\end{array}$ & 98 & $\begin{array}{c}98.8 \\
6\end{array}$ & $\begin{array}{c}98.8 \\
6\end{array}$ & $\begin{array}{c}98.8 \\
6\end{array}$ & $\begin{array}{c}99.1 \\
4\end{array}$ & $\begin{array}{c}99.4 \\
3\end{array}$ & $\begin{array}{c}99.1 \\
4\end{array}$ & $\begin{array}{c}99.1 \\
4\end{array}$ & $\begin{array}{c}98.8 \\
6\end{array}$ & $\begin{array}{c}98.5 \\
7\end{array}$ \\
\hline $\begin{array}{c}\text { Eucidea } \\
\text { n }\end{array}$ & $\begin{array}{c}965 \\
7\end{array}$ & $\begin{array}{c}965 \\
7\end{array}$ & $\begin{array}{c}96.5 \\
7\end{array}$ & $\begin{array}{c}96.5 \\
7\end{array}$ & $\begin{array}{c}965 \\
7\end{array}$ & $\begin{array}{c}96.5 \\
7\end{array}$ & $\begin{array}{c}96.5 \\
7\end{array}$ & $\begin{array}{c}965 \\
7\end{array}$ & $\begin{array}{c}96.5 \\
7\end{array}$ & $\begin{array}{c}96.5 \\
7\end{array}$ \\
\hline $\begin{array}{l}\text { Angle- } \\
\text { based }\end{array}$ & $\begin{array}{c}965 \\
7\end{array}$ & $\begin{array}{c}965 \\
7\end{array}$ & $\begin{array}{c}965 \\
7\end{array}$ & $\begin{array}{c}96.8 \\
6\end{array}$ & $\begin{array}{c}96.8 \\
6\end{array}$ & $\begin{array}{c}96.8 \\
6\end{array}$ & $\begin{array}{c}96.8 \\
6\end{array}$ & $\begin{array}{c}96.8 \\
6\end{array}$ & $\begin{array}{c}96.8 \\
6\end{array}$ & $\begin{array}{c}97.1 \\
4\end{array}$ \\
\hline
\end{tabular}
EQUAL TO 9

Some parameters in HOG are affecting its performance, such as the number of bins, cell size, and block size. To reach the optimal value of each parameter, we try to change one parameter while fixing some others. Starting with the bins number, Fig. 6 measures the performance of HOG at a different number of bins with different $\propto$ values for Modified Manhattan distance function, a cell size of 8 , and block size equal to 2 . It shows that the best accuracy ratio is acquired at the number of bins equals to 9 and 15 at $\propto$ equals to 0.05 , and 0.04 , respectively. It is a little disturbance to give the same accuracy ratio at different bin values at different $\propto$.

Fig. 7 and 8 introduces the effect of changing the number of bins on the HOG performance for the Manhattan distance function at different $\propto$ values, cell size $=8$, and block size $=2$. The results show that the Manhattan distance function has superior performance at $\propto=0.06$ and number of bins equal to 12 . We get an accuracy ratio of $100 \%$ at these parameters. 


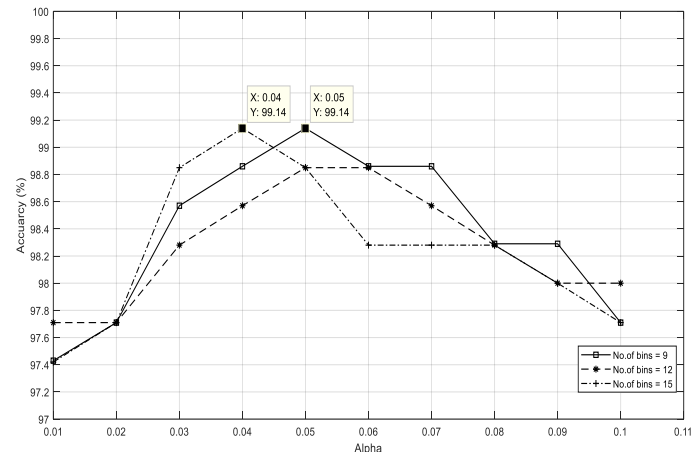

Fig. 6. Performance comparison of HOG at a different number of bins with different $\propto$ values for Modified Manhattan distance function.

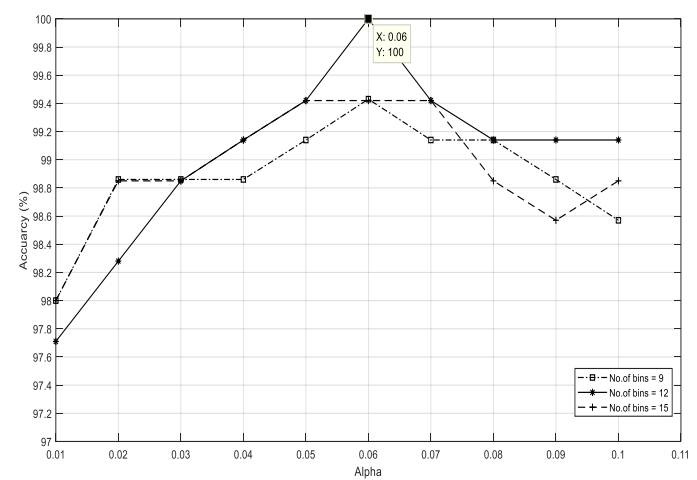

Fig. 7. Performance comparison of HOG at a different number of bins with different $\propto$ values for Manhattan distance function.

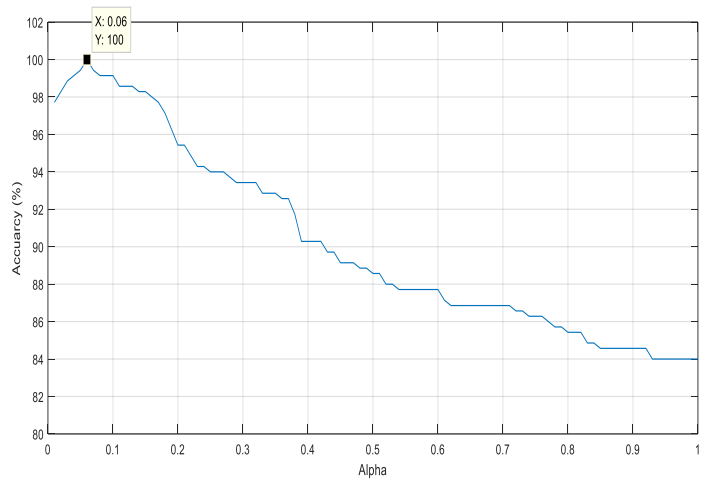

Fig. 8. Accuracy values of HOG at different values of $\propto$, and with number of bins equal to 12 for Manhattan distance function.

From this point up, we will use the Manhattan distance function as the best function matches feature vectors, with the number of bins equal to 12 and $\propto=0.06$. Now, the cell and block size is checked with the same number of bins and $\alpha$. Tables II and III show the effect of change the cell size and block size in the HOG performance, respectively. It is shown that the optimal number of cell size is equal to 8 , while the block size is 2 . The accuracy of individual identifying is still $100 \%$ at $\propto=0.06$ and a number of bins is equal to 12 .
TABLE. II. THE ACCURACY RATIOS IN PERCENTAGE FOR DIFFERENT CELL SIZES AND VARIOUS $\propto$ WITH A NUMBER OF HOG BINS EQUAL TO 12

\begin{tabular}{|c|c|c|c|c|c|c|c|c|c|c|}
\hline 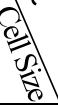 & 0.01 & 0.02 & $\mathbf{0 . 0 3}$ & 0.04 & 0.05 & 0.06 & 0.07 & 0.08 & 0.09 & 0.1 \\
\hline 4 & 96 & 96.57 & 96.57 & 96.85 & 97.71 & 98 & 97.42 & 98 & 98 & 98 \\
\hline 8 & 97.71 & 9828 & 98.85 & 99.14 & 99.42 & 100 & 99.42 & 99.14 & 99.14 & 99.14 \\
\hline 12 & 98.57 & 99.14 & 99.14 & 99.14 & 98.85 & 98.57 & 98.57 & 9828 & 98 & 97.71 \\
\hline 16 & 98.57 & 99.14 & 98.85 & 99.42 & 99.14 & 99.14 & 99.14 & 98.57 & 97.71 & 97.71 \\
\hline
\end{tabular}

TABLE. III. THE ACCURACY RATIOS IN PERCENTAGE FOR DIFFERENT BLOCK SIZES AND VARIOUS $\propto$ WITH A NUMBER OF HOG BINS EQUAL TO 12 AND CELL SIZE EQUAL TO 8

\begin{tabular}{|c|c|c|c|c|c|c|c|c|c|c|}
\hline 淧 & 0.01 & 0.02 & $\mathbf{0 . 0 3}$ & 0.04 & 0.05 & 0.06 & 0.07 & 0.08 & 0.09 & 0.1 \\
\hline 1 & 96 & 96 & 95.42 & 96 & 96 & 96 & 96 & 96 & 96 & 96.28 \\
\hline 2 & 97.71 & 9828 & 98.85 & 99.14 & 99.42 & 100 & 99.42 & 99.14 & 99.14 & 99.14 \\
\hline 4 & 98 & 99.42 & 99.42 & 99.42 & 99.42 & 99.71 & 99.71 & 99.71 & 98.85 & 98.57 \\
\hline 8 & 81.42 & 81.42 & 81.42 & 81.42 & 81.42 & 81.42 & 81.42 & 81.42 & 81.42 & 81.42 \\
\hline
\end{tabular}

Finally, in comparing the performance of face or digital signature features only with the merging of features between both of them as proposed, Fig. 9 shows the outperformance of multi-feature proposed method.

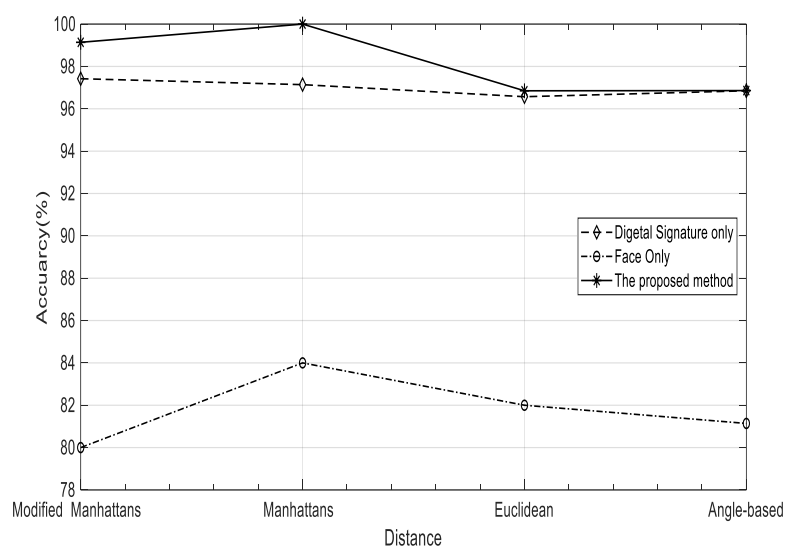

Fig. 9. Performance comparison of HOG at a different distance methods for face only, digital signature only, and the proposed method, at number of binsequal to 12 , and with $\propto=0.06$. 


\section{CONCLUSION}

This study presents a new hyperactive system that depends on HOG descriptor as features extraction for face recognition and digital signature together. Multi-biometric personal identification model using Histogram of Oriented Gradients (HOG) as feature extraction for face recognition and digital signature was present in this paper. The contributions are threefold: Firstly, to provide robustness to facial and signature feature detection, we propose to uniform sample the HOG as features. The result presented that our method performs better result for the multi biometrics system based on face recognition and digital signature instead of using them as an individual. Secondly, the matching result for our methods shows better result compared to other face recognition and digital signature only. This better performance is explained by the properties of HOG descriptor that is more robust for the hybrid. Finally, the results show that the HOG feature descriptor significantly performs target matching at an average of $100 \%$ accuracy ratio for face recognition together with the digital signature. It outperforms existing feature sets with an accuracy of $84.25 \%$ for face only and $97.42 \%$ for digital signature only. In near future, we hope to apply the deep-learning approaches for feature extraction instead to HOG. This way, we hope to gain a big range for Alpha $(\propto)$ selection with $100 \%$ accuracy.

\section{ACKNOWLEDGMENT}

This study has been supported by research support program, University of Bisha, Kingdom of Saudi Arabia, grand number (UB-06-1438).

\section{REFERENCES}

[1] Dalal, N. and B. Triggs. Histograms of oriented gradients for human detection. in Computer Vision and Pattern Recognition, 2005. CVPR 2005. IEEE Computer Society Conference on. 2005: IEEE.

[2] Rahmawati, E., et al. Digital signature on file using biometric fingerprint with fingerprint sensor on smartphone. in Engineering Technology and Applications (IES-ETA), 2017 International Electronics Symposium on. 2017: IEEE.

[3] Benaliouche, H. and M. Touahria, Comparative study of multimodal biometric recognition by fusion of iris and fingerprint. The Scientific World Journal, 2014. 2014.
[4] Jain, A.K., A. Ross, and S. Prabhakar, An introduction to biometric recognition. IEEE Transactions on circuits and systems for video technology, 2004. 14(1): p. 4-20.

[5] Kataria, A.N., et al. A survey of automated biometric authentication techniques. in Engineering (NUiCONE), 2013 Nirma University International Conference on. 2013: IEEE.

[6] Hernandez-Ardieta, J.L., et al., A taxonomy and survey of attacks on digital signatures. Computers \& security, 2013. 34: p. 67-112.

[7] Karaaba, M., et al. Robust face recognition by computing distances from multiple histograms of oriented gradients. in Computational Intelligence, 2015 IEEE Symposium Series on. 2015: IEEE.

[8] Lumini, A. and L. Nanni, Overview of the combination of biometric matchers. Information Fusion, 2017. 33: p. 71-85.

[9] Janbandhu, P.K. and M.Y. Siyal, Novel biometric digital signatures for Internet-based applications. Information Management \& Computer Security, 2001. 9(5): p. 205-212.

[10] Chelali, F.Z. and A. Djeradi. Zernike moments and histogram of oriented gradient descriptors for face recognition from video sequence. in Complex Systems (WCCS), 2014 Second World Conference on. 2014: IEEE.

[11] hihaoui, M., et al., A survey of $2 D$ face recognition techniques. Computers, 2016. 5(4): p. 21.

[12] Déniz, O., et al., Face recognition using histograms of oriented gradients. Pattern Recognition Letters, 2011. 32(12): p. 1598-1603.

[13] Tripathi, S.K. and B. Gupta, An Extension to Modified Harn Digital Signature Scheme with the Feature of Message Recovery, in Networking Communication and Data Knowledge Engineering. 2018, Springer. p. 183-193.

[14] Omara, I., et al. Discriminative Local Feature Fusion for Ear Recognition Problem. in Proceedings of the 2018 8th International Conference on Bioscience, Biochemistry and Bioinformatics. 2018: ACM.

[15] onnor, P. and A. Ross, Biometric recognition by gait: A survey of modalities and features. Computer Vision and Image Understanding, 2018. 167: p. 1-27.

[16] Lee, K. and M. Mokji. Automatic target detection in GPR images using Histogram of Oriented Gradients (HOG). in Electronic Design (ICED), 2014 2nd International Conference on. 2014: IEEE.

[17] Mathew, S. and G. Saranya. Advanced biometric home security system using digital signature and DNA cryptography. in Innovations in Green Energy and Healthcare Technologies (IGEHT), 2017 International Conference on. 2017: IEEE. 